\title{
NOUVELLE
}

\section{La bactérie Wolbachia bloque \\ l'infection des moustiques par différents pathogènes humains}

Marwan Naciri
École normale supérieure de Lyon, département de biologie, Master biologie, Lyon, France.
> Actuellement, de nombreux pathogènes émergent ou ré-émergent, principalement du fait du dérèglement climatique et de l'augmentation de la densité de population et des mobilités individuelles. C'est en particulier le cas des pathogènes transmis par les arthropodes hématophages et notamment par le moustique, dont certaines espèces tolèrent bien l'augmentation de température et s'adaptent efficacement aux environnements urbains. Les plus meurtriers de ces pathogènes sont le virus de la Dengue (DENV) et le virus Chikungunya (CHIKV), appartenant au groupe des arbovirus (arthropod-borne viruses), ainsi que le protozoaire Plasmodium, responsable du paludisme. Au total, ce sont plus de 500 millions de personnes qui sont infectées par ces pathogènes chaque année, dont plus de 700000 en meurent [1].

Une stratégie proposée pour lutter contre ces pathogènes consiste à diminuer la durée de vie des moustiques qui en sont les vecteurs. L'objectif est que celle-ci soit inférieure à la durée d'incubation minimale des pathogènes, c'est-à-dire la durée de développement minimale au sein du vecteur dont ont besoin les pathogènes avant qu'ils puissent être transmis à l'homme. Pour cela, il est possible d'introduire in natura des moustiques porteurs de certaines souches bactériennes du genre Wolbachia. Le genre Wolbachia regroupe des espèces de bactéries Gram-négatives qui sont associées à de très nombreux arthropodes. On estime en particulier que 40 à $60 \%$ des espèces d'insectes sont associées à Wolbachia. Lorsque Wolbachia infecte un organisme, elle pénètre dans les cellules et y persiste au sein de vésicules. Wolbachia infecte préférentiellement les cellules des organes sexuels, ce qui lui permet de manipuler la reproduction de son hôte de façon telle que la propagation de la bactérie au sein de la population hôte est favorisée. En laboratoire, il est possible d'infecter des moustiques par certaines souches de Wolbachia qui ne les infectent pas naturellement, comme par exemple des souches bactériennes isolées de drosophile. Contrairement aux infections qui surviennent naturellement, certaines de ces trans-infections provoquent la diminution de l'espérance de vie du moustique : introduire des moustiques porteurs d'une souche de Wolbachia adéquate est ainsi une stratégie envisagée pour lutter contre des pathogènes humains [2]. Moreira et al. ont étudié un autre effet sur le moustique de la trans-infection par Wolbachia: la modulation de sa résistance à l'infection par les pathogènes humains DENV, CHIKV et Plasmodium [3].

\section{La bactérie Wolbachia bloque \\ l'infection de son hôte par différents pathogènes}

Moreira et al. ont d'abord étudié l'impact de l'infection de moustiques avec la souche wMelPop de Wolbachia sur la capacité du DENV à les infecter. Des moustiques Aedes aegypti très répandus dans l'hémisphère sud et principaux vecteurs de nombreux virus dont le DENV et le CHIKV, issus d'élevage ou capturés, ont été infectés ou non par Wolbachia puis par le DENV. Ces expériences ont montré que la présence de Wolbachia est corrélée à un nombre de copies d'ARN du DENV jusqu'à $10^{4}$ fois plus faible par rapport aux moustiques non associés à la bactérie. Plus intéressant encore, chez les moustiques portant Wolbachia, aucune trace du virus n'était détectable dans la salive (par laquelle la transmission à l'homme a lieu lors d'une piqûre). Les auteurs ont en outre observé que chez les moustiques portant Wolbachia et infectés par le virus, bactérie et virus n'infectent pas les mêmes cellules.

Le même effet de Wolbachia contre une infection par le CHIKV et par Plasmodium a pu être mis en évidence. Le fait que Wolbachia interfère avec l'infection du moustique par des pathogènes aussi divers indique que le mécanisme d'action est probablement général.

\section{Comment la bactérie Wolbachia bloque-t-elle l'infection par ces pathogènes?}

Moreira et al. ont ensuite cherché à élucider les mécanismes responsables de ce blocage. Des travaux antérieurs avaient démontré que l'expression de gènes de l'immunité de A. aegypti est activée lors d'une infection par le DENV. De même, le microbiote intestinal du moustique peut également activer l'expression de certains de ces gènes [4]. Les auteurs ont donc avancé I'hypothèse que l'activation du système immunitaire du moustique par Wolbachia permet à celui-ci de lutter plus efficacement contre une infection ultérieure par un autre pathogène. Ils ont donc étudié par RT-qPCR (reverse transcription quantitative polymerase chain reaction) le taux d'expression de certains de ces gènes à l'échelle de l'organisme 
entier, en présence ou non de Wolbachia. Ils ont ainsi démontré que Wolbachia a pour effet d'activer l'expression de gènes impliqués dans la lutte antimicrobienne, dont les gènes codant des peptides antimicrobiens comme les cécropines, les défensines et les lectines de type $C$, ce qui en retour pourrait limiter la propagation d'autres microorganismes, comme les arbovirus et Plasmodium étudiés ici.

Les auteurs de cette étude suggèrent également que l'inhibition de l'infection par le DENV par Wolbachia peut être due, en partie du moins, à une compétition entre les deux pathogènes pour certains composants cellulaires. Le cholestérol de l'hôte est un candidat sérieux puisqu'il est nécessaire à la survie de nombreuses bactéries parasites [5] ainsi qu'à l'infection, la réplication et la transmission de certains arbovirus $[6,7]$ et au développement de Plasmodium au sein de son hôte [8]. Cela serait cohérent avec l'exclusion réciproque de Wolbachia et du DENV à l'échelle cellulaire observée par les auteurs de cette étude. Dans des travaux plus récents, plusieurs équipes ont confirmé cette hypothèse et ont démontré que des lipides, en particulier le cholestérol, jouent en effet un rôle dans le blocage de la réplication du DENV $[9,10]$. Geoghegan et al. ont notamment montré que Wolbachia perturbe le métabolisme et le transport du cholestérol dans les cellules de moustique, et que lorsque l'homéostasie du cholestérol est restaurée grâce à une drogue, le DENV retrouve sa capacité de réplication habituelle [9]. Ces découvertes n'excluent pas la possibilité que l'activation du système immunitaire par les bactéries en amont de l'infec- tion virale joue également un rôle dans ce blocage mais les résultats concernant cette hypothèse sont contradictoires. Alors que certaines équipes rapportent la nécessité de cette préactivation du systéme immunitaire [1], 12], d'autres observent qu'elle n'est pas indispensable au blocage de la réplication virale [13].

Plusieurs études (dont la première de Moreira et al.) démontrent que l'effet de Wolbachia sur la réduction de l'infection par un autre pathogène est d'autant plus fort que la densité de la bactérie au sein de l'hôte moustique est importante $[14,15]$. Or, la densité de Wolbachia d'une souche donnée tend à être plus importante au sein d'hôtes qui ne sont pas infectés naturellement par cette souche. L'ensemble de ces découvertes indique donc que, pour exploiter au mieux ce phénomène d'interférence entre l'infection bactérienne et l'infection par des pathogènes humains dans le cadre de la lutte contre la transmission de ces derniers, il faudrait introduire des moustiques parasités par une souche de Wolbachia ne les infectant pas naturellement. L'identification de souches de Wolbachia présentant une très bonne efficacité d'inhibition de l'infection par des pathogènes humains et une capacité de propagation importante au sein des populations de moustiques constitue actuellement un défi pour la communauté scientifique. $\diamond$

\section{Wolbachia bacteria inhibits mosquito} infection by various human pathogens

\section{LIENS D'INTÉRÊT}

L'auteur déclare n'avoir aucun lien d'intérêt concernant les données publiées dans cet article.

\section{RÉFÉRENCES}

1. World Health Organization. Vector-borne diseases 2017. https://www.who.int/news-room/fact-sheets/ detail/vector-borne-diseases.

2. Brownstein JS, Hett $\varepsilon$, O'Neill SL. The potential of virulent Wolbachia to modulate disease transmission by insects. J Invertebr Pathol $2003 ; 84: 24-9$.

3. Moreira LA, Iturbe-Ormaetxe I, Jeffery JA, et al. A Wolbachia symbiont in Aedes aegypti limits infection with Dengue, Chikungunya, and Plasmodium. Cell 2009 ; 139 : 1268-78.

4. Xi Z, Ramirez JL, Dimopoulos G. The Aedes aegypti toll pathway controls Dengue virus infection. PLoS Pathog 2008 ; 4 : e1000098.

5. Lin M, Rikihisa Y. Ehrlichia chaffeensis and Anaplasma phagocytophilum lack genes for lipid A biosynthesis and incorporate cholesterol for their survival. Infect Immun $2003 ; 71: 5324-31$.

6. Gay B, Bernard E, Solignat M, et al. pH-dependent entry of chikungunya virus into Aedes albopictus cells. Infect Genet Evol 2012 ; 12 : 1275-81.

7. Carro AC, Damonte EB. Requirement of cholesterol in the viral envelope for dengue virus infection. Virus Res $2013 ; 174: 78-87$.

8. Atella GC, Bittencourt-Cunha PR, Nunes RD, et al. The major insect lipoprotein is a lipid source to mosquito stages of malaria parasite. Acta Trop 2009 ; 109 : 159-62.

9. Geoghegan V, Stainton K, Rainey SM, et al. Perturbed cholesterol and vesicular trafficking associated with dengue blocking in Wolbachia-infected Aedes aegypti cells. Nat Commun $2017 ; 8: 526$.

10. Caragata $\varepsilon P$, Rancès $\varepsilon$, Hedges LM, et al. Dietary cholesterol modulates pathogen blocking by Wolbachia. PLoS Pathog 2013 ; 9 : el003459.

11. Kambris Z, Blagborough AM, Pinto SB, et al. Wolbachia stimulates immune gene expression and inhibits Plasmodium development in Anopheles gambiae. PLoS Pathog 2010 ; 6 : el001143.

12. Pan $X$, Zhou G, Wu J, et al. Wolbachia induces reactive oxygen species (ROS)-dependent activation of the Toll pathway to control dengue virus in the mosquito Aedes aegypti. Proc Natl Acad Sci USA 2012 ; 109 : ع23-31.

13. Rancès $\varepsilon$, Johnson TK, Popovici J, et al. The Toll and Imd pathways are not required for Wolbachiamediated Dengue virus interference. J Virol 2013 ; 87 $11945-9$.

14. Lu P, Bian G, Pan X, Xi Z. Wolbachia induces densitydependent inhibition to Dengue virus in mosquito cells. PLoS Negl Trop Dis $2012 ; 6$ : el754.

15. Bian G, Zhou G, Lu P, Xi Z. Replacing a native Wolbachia with a novel strain results in an increase in endosymbiont load and resistance to Dengue virus in a mosquito vector. PloS Negl Trop Dis 2013 ; 7 : e2250. 